\title{
Violência contra professores nas escolas brasileiras: determinantes e consequências
}

\author{
Violence against teachers in Brazilian schools: determinants and consequences
}

\author{
Wander Plassa (1) \\ Pietro André Telatin Paschoalino(2) \\ Luan Vinicius Bernardelli (1) \\ (1) Universidade Estadual do Paraná \\ (2) Universidade Estadual de Maringá
}

\begin{abstract}
This study aims to evaluate how the sociodemographic characteristics and the school environment affect the probability of violence toward the teacher. In a complementary way, we investigate the impact of school violence on content developed in class, variable related to students' learning. We use data from the National System for the Evaluation of Basic Education in 2017 and the multinominal Logit and Logit models. The results show that teachers' personal characteristics such as being a man, older, and receiving low salary increase the likelihood of reports of physical violence. In addition, teachers who teach in classes made up of a majority of non-white, male, single-parent families and low mother education students are more likely to report violence. It is also worth mentioning that reporting being a victim, whether physical or against property, considerably decreases the chances of teachers developing the programmed content.
\end{abstract}

\section{Keywords}

school violence, teachers, work environment.

JEL Codes I29, C25, K42.

\section{Resumo}

Este artigo objetiva avaliar como as características sociodemográficas e do ambiente escolar afetam a probabilidade de violência contra o professor. De forma complementar, investiga o impacto da violência escolar sobre o conteúdo desenvolvido em classe, variável relacionada ao aprendizado dos alunos. Foram utilizados os dados do Sistema Nacional de Avaliação da Educação Básica (SAEB) de 2017, aliados aos modelos Logit e Logit multinominal. Os resultados mostram que características pessoais dos professores como ser homem, mais velhos e receber baixo salário aumentam a probabilidade de relatos de violência contra a vida. Além disso, professores que lecionam em classes formadas por maioria de alunos homens, não brancos, de familias monoparentais e com educação materna baixa possuem maiores chances de relatar violência. Cabe destacar ainda que sofrer violência, seja ela física ou contra propriedade, diminui consideravelmente as chances de os professores desenvolverem o conteúdo programado.

\section{Palavras-chave}

violência escolar, professores, ambiente de trabalho.

Códigos JEL I29, C25, K42. 


\section{Introdução}

O professor é um dos principais atores dentro do processo de aprendizagem, que além de ministrar o conteúdo em sala de aula, também contribui na motivação e orientação, agindo como facilitador de aprendizagem de seus alunos (Duarte, 2009; Silva; Navarro, 2012). Para que esses profissionais possam exercer plenamente suas atividades, no entanto, é necessário um ambiente escolar seguro, tanto para eles quanto para seus alunos.

Todavia, no Brasil contemporâneo, a violência no âmbito escolar está entre os principais problemas enfrentados pelas escolas, gerando consequências negativas sobre os resultados escolares dos alunos e do próprio professor (Becker; Kassouf, 2016; Monteiro; Rocha, 2017; Silva; Rosa, 2013). Pesquisas recentes em âmbito internacional e nacional destacam a gravidade do problema, especialmente no Brasil, um dos países com maior índice de violência contra o professor (OCDE, 2013; SAEB/Prova Brasil, 2017).

Mesmo diante da importância do professor no processo de aprendizado, das altas taxas de relatos de violência sobre eles e das possíveis consequências que essa violência pode acarretar nas rotinas desses profissionais, Galand et al. (2007) relatam que trabalhos analisando os impactos da violência contra os professores têm sido relativamente negligenciados na literatura. No Brasil, Tavares e Pietrobom (2016) destacam a carência de trabalhos com tal objetivo. O que se nota, em geral, são trabalhos descritivos e de estudos de casos em municípios específicos.

Portanto, visto a relativa escassez de estudos e a necessidade de avançar no debate para que as políticas públicas possam ser eficazes, o presente estudo tem por objetivo avaliar quais são as características que influenciam a probabilidade de o professor relatar ter sido vítima de violência no ambiente escolar. Além disso, pautado na conclusão de Wilson et al. (2011), de que a recente literatura centra-se na prevalência da violência e não em suas consequências sobre o professor, na segunda parte deste artigo é avaliado o efeito dessa violência sobre o desempenho desse profissional (captado pelo conteúdo desenvolvido em classe).

O trabalho busca contribuir para o debate ainda incipiente sobre violência dirigida aos professores, temática que é especialmente importante para o Brasil, país em que o problema parece apresentar grandes proporções. Além disso, visto que os relatos de violência nas escolas brasileiras ocorrem em diferentes regiões, utilizam-se dados em nível nacional extraídos 
do Sistema Nacional de Avaliação da Educação Básica (SAEB), o que é pouco explorado pela escassa literatura nacional.

Para cumprir os objetivos estabelecidos nesta introdução, o presente trabalho está dividido em mais quatro seções. A segunda seção trata da revisão de literatura sobre o tema do estudo. A seção posterior trata da metodologia empregada, assim como da base de dados utilizada. A quarta seção analisa e discute os resultados encontrados. Por fim, na última seção, apresentam-se as conclusões do estudo.

\section{Revisão bibliográfica}

A violência observada no meio escolar pode ter consequências sérias sobre aqueles que fazem parte do seu cotidiano e do desempenho do processo educativo. Segundo Espelage et al. (2013), a violência sobre os professores tem sido associada a aumento da insegurança, a sintomas físicos e emocionais, a níveis elevados de estresse, a relacionamentos pessoais deteriorados, assim como desempenho insatisfatório no trabalho. Elliott et al. (1998) destacam que não é surpreendente identificar na literatura resultados caminhando nesse sentido. A violência sobre o professor pode acarretar em menor motivação e comprometimento (Vettenburg, 2002), maior ausência e maior rotatividade (Monteiro; Rocha, 2017; Duarte, 2009), em maior propensão a abandonar a profissão (Newman et al., 2008) e, para a escola, dificuldade de recrutamento e na retenção de profissionais altamente eficazes (Espelage et al., 2013).

O quadro se torna ainda mais grave ao se observar grande incidência de relatos de violência contra os professores. Longobardi et al. (2018), realizando uma revisão sistemática e abrangente envolvendo 24 estudos, encontraram evidências da alta taxa de violência direcionada aos professores, especialmente quando se trata de formas físicas e não físicas de violência. Estudos de casos realizados no Brasil mostram que essa também é a tendência em escolas do ensino fundamental e médio.

De acordo com Carvalho et al. (2016), em um estudo considerando 48 docentes em duas escolas do ensino fundamental do munícipio de Simões (PI), verificou-se que a maior parte deles afirmaram já ter observado violência na escola em que 55,1\% relataram ter sido eles próprios vítimas dessa violência. Rocha et al. (2012), com dados de 121 profissionais de 
duas escolas, uma particular e uma pública, da cidade de Natal (RN), encontraram que dos 62 funcionários da escola pública e 59 da escola privada amostrados, $43,5 \%$ e $25,0 \%$ foram vítimas de algum tipo de violência dentro do ambiente escolar, respectivamente. Dessa forma, é possível que o problema seja ainda mais grave nas instituições públicas.

Até mesmo os tipos mais graves de violência contra o professor, como tentativas ou agressões físicas, atingem um percentual relevante de casos. Foi o que constataram Melanda et al. (2018) ao analisarem 784 docentes dos ensinos fundamental e médio de 20 escolas estaduais da cidade de Londrina (PR). Do total de professores analisados, 8,4\%, ou seja, um em cada 12 professores, relatou ter sido vítima de violência escolar, em alguns casos, envolvendo até mesmo o uso de arma de fogo (quatro casos) ou armas brancas (seis casos), nos 12 meses anteriores à pesquisa. Além disso, características sociodemográficas, principalmente relacionadas à condição de trabalho do professor, apresentaram associação significativa com a violência física.

Diante desse quadro grave de violência contra o professor no Brasil, o maior entendimento da violência sobre o professor no seu local de trabalho pode, através de ações efetivas, ajudar promover a melhoria do bem-estar desses profissionais, beneficiando seus estudantes (Longobardi et al., 2018). Além disso, a compreensão dos fatores relacionados à violência escolar é fundamental para melhoria na capacidade de detecção de tais comportamentos, uma vez que a escola tem um papel relevante na identificação do indivíduo com tendência a apresentar comportamento violento, já que se trata de um ambiente em que normalmente é manifestado tal comportamento (Becker; Kassouf, 2016).

Diversos estudos têm tentado localizar os determinantes da violência escolar, como o estudo realizado por Gerberich et al. (2011). Os autores buscaram identificar a magnitude e os fatores de risco para agressão física ocupacional e violência não física contra educadores de Minnesota, nos Estados Unidos, através de uma amostra aleatória inicial de 26.000 educadores de ensinos fundamental e médio desde 2003. Para isso, eles utilizaram modelos lineares generalizados a fim de calcular as razões de incidência de agressão física e violência não física contra os educadores, assim como odds ratio a partir de regressão logística. O estudo ainda avançou no sentido de analisar o perfil dos agressores, que eram em sua grande maioria estudantes com incapacidade ou atraso no desenvolvimento. 
Os resultados de Gerberich et al. (2011) ainda apontam que as mulheres tendem a apresentar mais relatos de vitimização da violência escolar. Por sua vez, da análise multivariada, encontram-se riscos reduzidos em ambos os tipos de violência para homens, em comparação com mulheres, assim como os resultados mostram que a experiência do educador é um fator de proteção contra a violência escolar.

Um trabalho correlato realizado por Ibabe et al. (2013) objetivou analisar o papel da família como fator de proteção para a violência contra a autoridade (pais e professores). Os autores analisaram cinco escolas urbanas em Gipuzkoa, na Espanha, levando em consideração o tipo de escola (pública ou privada) e o idioma ministrado. Um total de 687 estudantes entre 12 e 16 anos foi avaliado. Os resultados mostram que um ambiente familiar positivo, mensurado por meio das percepções das pessoas sobre seus ambientes familiares, é um dos fatores mais importantes contra o comportamento violento, seguindo resultados similares aos de Shochet et al. (2008) e Jaureguizar et al. (2013).

Outro estudo que aponta a importância das características dos professores foi conduzido por Huang et al. (2017) e avaliou 24.070 professores em 4.610 escolas nos Estados Unidos durante os anos de 2011 e 2012. Para os autores, professores mais experientes têm menos probabilidade de relatar ameaças ou ataques físicos, comparativamente a professores menos experientes. Adicionalmente, eles observaram que a aplicação de regras estabelecidas pelos diretores com apoio do corpo administrativo atua positivamente contra a violência escolar.

Outra importante pesquisa é a realizada por Chen e Astor (2008), os quais utilizam uma amostra com mais de 14.000 estudantes em Taiwan, dos ensinos fundamental e médio. Para quantificar a violência contra os professores, os alunos classificaram sete tipos de violência codificados em "nunca" e "pelo menos uma vez", em que, para cada tipo de violência, foram fornecidas nove razões para tal.

Ainda que de forma descritiva, os autores mostram que, no geral, $30,1 \%$ dos estudantes de Taiwan relataram envolvimento em pelo menos um ato agressivo contra seus professores durante o ano anterior à pesquisa. Além disso, destaca-se que a taxa de perpetração de cometer ao menos uma vez violência é maior para alunos masculinos em relação aos femininos (Chen; Astor, 2008). 
O estudo exploratório de Lowe et al. (2020) analisou a violência direcionada à professores do ensino fundamental e médio por estudantes de uma região da Austrália entre os anos de 2016 e 2018. Os professores foram recrutados por conveniência e amostragem intencional. Uma amostra de conveniência de cinco participantes foi recrutada da reunião geral anual do Conselho Australiano de Saúde, Educação Física e Recreação e uma técnica de amostragem não probabilística proposital, através das mídias sociais, foi responsável por recrutar 51 professores da Austrália Ocidental de uma população de 35.012 .

Os autores utilizaram o modelo linear generalizado para categorias de violência e características demográficas para prever a violência direcionada aos professores. Os autores encontraram que, as chances de serem intimidados ou ameaçados eram cinco vezes maiores de ocorrer se o participante fosse do sexo feminino, controlando o código postal (índices socioeconômicos para áreas da escola) e setor (escola pública ou privada). Trabalhar numa escola pública (governo) localizada num índice socioeconômico baixo para áreas da escola (menor que percentil de 74\%) aumentou cinco vezes as chances de um professor experimentar gestos obscenos e roubo de bens pessoais.

Portanto, nota-se que os estudos que analisam os determinantes da violência no ambiente escolar já foram realizados em diversos contextos. Os resultados, em geral, apontam que variáveis como sexo e experiência do professor, a aplicação ou não das regras no ambiente escolar, localização e sua relação com índices socioeconômicos, assim como características da sala de aula (número de alunos homens ou mulheres e sua estrutura familiar) são fatores importantes na explicação desse fenômeno.

\section{Metodologia}

\subsection{Base de dados}

São utilizadas informações disponibilizadas pelo Instituto Nacional de Estudos e Pesquisas Educacionais Anísio Teixeira (INEP) através do levantamento da Prova Brasil. A Prova Brasil é a uma avaliação censitária de alunos da $4^{\mathrm{a}}$ série $/ 5^{\circ}$ ano, $8^{\mathrm{a}}$ série $/ 9^{\circ}$ ano do Ensino Fundamental e $3^{\circ}$ ano do Ensino Médio das escolas públicas das redes municipal, estadual e fe- 
deral. Nessa base são encontrados resultados em testes padronizados de proficiência em português e matemática, suas características demográficas e seu background socioeconômico. Além disso, há informações sobre as características estruturais das escolas e percepção de violência local por parte dos diretores e professores.

Para alcançar o objetivo deste artigo, selecionou-se o ano de 2017, o mais recente da pesquisa. Em 2017, a Prova Brasil abordou um total de 6.931.985 alunos, distribuídos em 73.674 escolas. As características desses alunos e das escolas foram abordadas, conforme a Tabela A1 e conectadas aos docentes. Professores que ministravam aulas em escolas diferentes e turmas que se repetiam na amostra foram retirados da análise. Esse procedimento foi realizado com o intuito de identificar precisamente o efeito turma e escola sobre o relato de vitimização do professor. Também foram retirados das análises os profissionais que não responderam às perguntas de interesse nessa pesquisa, como violência e características do profissional.

Por fim, foram mantidos apenas os profissionais que lecionavam em áreas urbanas, uma vez que o fenômeno estudado é mais persistente nessas áreas (Glaeser; Sacerdote, 1999) e em escolas estaduais e municipais, uma vez que em escolas privadas a violência tende a ser menor (Lowe et al., 2020; Carvalho et al., 2016). O número total de professores após os filtros realizados foi de 38.263 .

\subsection{Métodos econométricos}

Diante da natureza das variáveis de interesse, dois métodos econométricos qualitativos são propostos. O primeiro, Logit, é amplamente utilizado quando se pretende analisar características que impactam a probabilidade de um evento binário ocorrer. Por exemplo, Becker e Kassouf (2016) utilizaram o mesmo método para investigar os fatores associados à chance de se observar (ou não) violência cometida por alunos do ensino fundamental no ambiente escolar do Brasil para os anos de 2007 e 2009.

No presente estudo, além das próprias características do professor, variáveis relacionadas ao ambiente da sala (captadas pelas características dos alunos) e ambiente escolar (captadas por meio das características da escola e do diretor) são consideradas. Nessa primeira parte, o evento qualitativo 
analisado é o relato, por parte do docente, de ser vítima ou não de dois tipos de crimes no ambiente escolar: a) crime contra a vida (atentado contra a vida ou ameaça); e, b) crime contra a propriedade (furto ou roubo). São estimadas duas especificações, uma para cada tipo de crime, em que se calcula a probabilidade de o professor $i$ relatar atos de violência $\left(y_{i}=1\right)$ ou não $\left(y_{i}=0\right)$ dadas as suas características $\left(\boldsymbol{x}_{i}\right)$, conforme a equação (1):

$$
\operatorname{Pr}\left[y_{i}=1 \mid \boldsymbol{x}_{i}\right]=\frac{\exp \left[\boldsymbol{x}_{i}^{\prime} \beta\right]}{1+\exp \left[\boldsymbol{x}_{i}^{\prime} \beta\right]}=\Lambda\left[\boldsymbol{x}_{i}^{\prime} \beta\right]
$$

A notação $\Lambda($.) é utilizada para indicar a função de distribuição acumulada logística. Conforme Cameron e Trivedi (2005), há uma interpretação comum da literatura dos efeitos em termos das razões de chances. Assim, considerando $p=\operatorname{Pr}\left[y_{i}=1 \mid \boldsymbol{x}_{i}\right]$ a razão de chances dada por $p /(1-p)$ mensura a probabilidade de $y_{i}=1$ relativa à probabilidade de $y_{i}=0$. Com esse procedimento, subtraindo 1 dos valores dos coeficientes estimados da regressão, encontram-se os valores de interesse. No caso de valores positivos um evento tem maior probabilidade de ocorrer e, para valores negativos, um evento tem menor probabilidade de se realizar.

Na sequência à estimação do método Logit o objetivo é verificar a probabilidade que um professor tem em ministrar menos de $60 \%$, entre $60 \%$ e $80 \%$ ou mais de $80 \%$ do conteúdo estipulado para o ano letivo dadas as suas características $\left(\boldsymbol{x}_{i}\right)$. Nessa segunda estimação, as variáveis de exposição à violência, utilizadas como dependentes na estimação Logit, são consideradas como explicativas, porquanto se espera que elas sejam associadas a menor capacidade de ministrar o conteúdo proposto pelo professor (Vettenburg, 2002; Espelage et al., 2013).

Como nesse caso há mais de duas possibilidades, sem ordenação clara e as alternativas são mutuamente exclusivas, propõe-se o método Logit Multinomial (Cameron; Trivedi, 2005). Um exemplo de aplicação desse método pode ser visto no trabalho de Miller et al. (1999). Os autores analisaram os fatores que contribuíam para que um professor, do ensino fundamental ou médio, permanecesse, deixasse ou se transferisse de um determinado tipo especial de escola nos Estados Unidos.

Seja então $y_{i}$ uma variável aleatória, em que $y_{i} \in\{0,1,2\}$ e $\boldsymbol{x}_{i}$ denota um conjunto de variáveis condicionais, a equação (2) pode ser apresentada como: 


$$
p_{i j}=\operatorname{Pr}\left[y_{i}=j \mid \boldsymbol{x}_{i}\right]=\frac{\exp \left[\boldsymbol{x}_{i}^{\prime} \boldsymbol{\beta}_{j}\right]}{\sum_{k=0}^{2} \exp \left[\boldsymbol{x}_{i}^{\prime} \beta_{j}\right]}, \quad j=0,1 e 2,
$$

o subscrito $i$ denota o indivíduo (professor) e subscrito $j$ ou $k$ a alternativa. Como $\sum_{j=0}^{2} p_{i j}=1$, para garantir a identificação do modelo uma restrição do tipo $\beta_{j}=0$ é necessária. Neste estudo, é definido $\beta_{0}=0$, isto é, a alternativa "menos de $60 \%$ de conteúdo desenvolvido" é normalizada para zero, sendo ela o grupo de referência na interpretação dos resultados. Portanto, segundo Greene (2011), com uma das alternativas normalizadas, pode-se obter a equação (3):

$$
p_{i j}=\operatorname{Pr}\left[y_{i}=j \mid \boldsymbol{x}_{i}\right]=\frac{\exp \left[\boldsymbol{x}_{i}^{\prime} \beta_{j}\right]}{1+\sum_{k=1}^{2} \exp \left[\boldsymbol{x}_{i}^{\prime} \beta_{j}\right]}, \quad j=0,1 e 2,
$$

No caso do Logit Multinomial é possível apresentar os resultados em termos de Relação de Risco Relativo (RRR), facilitando a interpretação. Eles podem ser obtidos através da exponencial dos coeficientes do Logit Multinomial, $\exp [\hat{\beta}]$. A RRR de um coeficiente estimado indica como o risco de cair em um grupo comparado ao risco de cair no grupo de referência se altera com a variável em questão. Isto é, um $R R R>1$ indica que o risco de o resultado cair no grupo em questão em relação ao risco de o resultado cair no grupo de referência se amplia à medida que a variável analisada aumenta, por exemplo.

\section{Resultados e discussão}

\subsection{Análise descritiva}

A Tabela 1 apresenta a média e o desvio padrão das variáveis utilizadas no presente estudo para a amostra geral e para os recortes de grupos de vitimização dos professores. Da amostra geral, com 38.263 professores, $5,27 \%(n=2.018)$ relataram ter sofrido crimes contra propriedade, enquanto $8,24 \%(n=3.152)$ relataram terem sofrido violência contra a vida nas dependências da escola no último ano. Destaca-se que a maior parte dos docentes da amostra são mulheres (78,5\%) e não brancos (53,5\%). 
Tabela 1 Características gerais dos professores por grupos de vitimização no Brasil, 2017

\begin{tabular}{l|r|r|r|r|r|r}
\hline \multirow{2}{*}{ Variáveis } & Média & D.P. & Média & D.P. & Média & D.P. \\
\cline { 2 - 7 } & & Geral & & Contra a vida & Contra a propriedade \\
\hline Mulher & 78,52 & 41,07 & 70,40 & 45,66 & 78,99 & 40,75 \\
\hline Não branco & 53,50 & 49,88 & 53,33 & 49,90 & 56,05 & 49,65 \\
\hline Maior que graduação & 70,37 & 45,66 & 69,42 & 46,08 & 70,71 & 45,52 \\
\hline Maior que 4 salários mínimos & 26,61 & 44,19 & 26,62 & 44,20 & 28,30 & 45,05 \\
\hline Carga horária superior a 40 horas & 40,47 & 49,08 & 39,88 & 48,97 & 43,61 & 49,60 \\
\hline Experiência superior a 2 anos & 96,48 & 18,42 & 96,45 & 18,52 & 97,13 & 16,71 \\
\hline Aplicação de normas & 88,78 & 31,56 & 82,61 & 37,90 & 83,40 & 37,22 \\
\hline Ambiente desestruturado & 13,56 & 34,23 & 41,18 & 49,22 & 35,58 & 47,89 \\
\hline Ambiente violento & 5,27 & 22,35 & 23,60 & 42,47 & 22,55 & 41,80 \\
\hline No de alunos na sala & 27,79 & 7,02 & 28,17 & 7,32 & 28,62 & 7,07 \\
\hline Amostra & 38.263 & $(100 \%)$ & 3.152 & $(8,24 \%)$ & 2.018 & $(5,27 \%)$ \\
\hline
\end{tabular}

Fonte: Elaboração própria, com base nos dados da Prova Brasil (2017).

Em relação à escolaridade dos professores, verifica-se que a maioria, detinha pós-graduação (latu sensu com mais de 360 horas ou mestrado/ doutorado). Por sua vez, quando analisada a variável renda, pouco mais de um quarto da amostra recebia mais de 4 salários mínimos, ou seja, a maior parte dos professores recebiam até $\mathrm{R} \$ 3.748,00$ (a preços correntes de 2017).

Com relação à carga horária do professor pode se observar que aproximadamente $40,5 \%$ da amostra trabalhava mais de 40 horas, o que pode, por um lado, ser um importante fator para aumento do rendimento do trabalho, mas que, por outro, pode levar a maiores níveis de estresse. Por sua vez, de acordo com os valores apresentados para a experiência, aproximadamente $96,5 \%$ dos professores tinham mais de dois anos de experiência como professor. Destaca-se que a experiência dos professores é uma importante variável explicativa, uma vez que de acordo com a literatura quanto maior a experiência menor a probabilidade de o professor ser atacado (Huang et al., 2017).

Verifica-se, ainda, através da variável "Desestruturada" e "Violenta", que no ambiente escolar a segurança do professor e mesmo dos alunos pode ser questionável. Identificou-se que aproximadamente 13,6\% dos professores relataram escolas desestruturadas (com a presença de alunos sob efeito de drogas e álcool), e 5,3\%, escolas violentas (com a presença 
de alunos com armas). Quando avaliados por recortes de violência, esses valores aumentam consideravelmente.

A Tabela 2 mostra as características do ambiente em sala de aula, através das características dos alunos, apresentados para a amostra geral e pelos grupos de vitimização. Foram considerados 893.318 alunos, em que $72.195(8,08 \%)$ eram alunos de professores que já relataram ser vítimas de crimes contra a vida, e 47.372 (5,30\%), de professores que relataram ser vítimas de crime contra propriedade. Do total de alunos, aproximadamente, $49 \%$ eram homens e, por sua vez, $66 \%$ dos alunos eram não brancos.

Tabela 2 Características gerais dos alunos por grupos de vitimização de seus professores no Brasil, 2017

\begin{tabular}{l|r|r|rr|r|r}
\hline Variáveis & Média & D.P. & Média & D.P. & Média & D.P. \\
\cline { 2 - 7 } & & Geral & \multicolumn{3}{c}{ Contra a vida } & Contra a propriedade \\
\hline Homens & 49,19 & 49,99 & 49,54 & 50,00 & 50,08 & 50,00 \\
\hline Não branco & 66,08 & 47,34 & 67,07 & 47,00 & 67,53 & 46,83 \\
\hline Reside com pais & 62,06 & 48,52 & 59,35 & 49,12 & 59,69 & 49,05 \\
\hline Ensino médio ou superior (mãe) & 47,77 & 49,95 & 45,60 & 49,81 & 48,10 & 49,96 \\
\hline Reprovação & 26,62 & 44,20 & 30,17 & 45,90 & 27,87 & 44,84 \\
\hline Abandono & 2,90 & 16,78 & 3,24 & 17,70 & 3,34 & 17,97 \\
\hline Amostra & 893.318 & $(100 \%)$ & 72.195 & $(8,08 \%)$ & 47.372 & $(5,30 \%)$ \\
\hline
\end{tabular}

Fonte: Elaboração própria, com base nos dados da Prova Brasil (2017).

$\mathrm{Na}$ amostra geral, $62 \%$ dos alunos residiam com ambos os pais. Porém, considerando os recortes de vitimização, tal valor diminui para 59,35\% e $59,69 \%$, respectivamente, em crimes contra a vida e em crimes contra propriedade. Outra informação importante de background familiar é a proporção de alunos que possuíam mães com ao menos ensino médio completo. Como se observa, apenas $48 \%$ das mães detêm tal escolaridade. Essas duas variáveis podem estar relacionadas com diversas questões familiares e de apoio aos estudantes. Como mostrado no referencial teórico sobre o tema, o ambiente familiar é deveras importante contra o comportamento violento (Ibabe et al., 2013).

Verifica-se, ainda, que $26,62 \%$ dos alunos já haviam sido reprovados pelo menos uma vez. Além disso, observa-se que esse percentual aumenta nos recortes de vitimização dos professores. Em relação ao abandono, $2,9 \%$ dos alunos relataram ter abandonado a escola pelo menos uma vez. 
Tendo discorrido sobre as principais questões da amostra, a próxima subseção apresentará os resultados econométricos, buscando averiguar os fatores que impactam a probabilidade de o professor ser vítima na escola, assim como o impacto de tais crimes sobre o conteúdo desenvolvido em sala.

\subsection{Resultados econométricos}

A Tabela 3 apresenta a estimação do modelo Logit com os coeficientes representados pelas razões de chances em quatro especificações. Os resultados foram divididos entre crimes contra a vida e de crime contra a propriedade, utilizando duas subdivisões, uma para os controles adicionais, de características dos alunos e da escola, e outra que não leva esses controles em consideração.

No primeiro grupo, analisam-se as características dos professores, tais como sexo, cor de pele, escolaridade, rendimento, experiência e carga horária trabalhada. Os resultados indicam que o fato de o professor ser mulher impacta negativamente a probabilidade de relatos de crimes contra a vida. No caso de relatos de crimes contra propriedade em âmbito escolar, o sentido do coeficiente é positivo. É importante mencionar que conforme abordado na seção 2 deste artigo, não há um consenso na literatura em relação ao impacto do sexo na percepção de violência no ambiente escolar (Gerberich et al., 2011; Lowe et al., 2020). Dessa forma, essa divergência de resultados encontrada na literatura pode advir de diferentes métricas de violência.

Um resultado interessante a ser analisado é o nível educacional do professor que, em princípio, pode influenciar a ocorrência de violência nas escolas, uma vez que a formação e o conhecimento tendem a estar associados com a capacidade de lidar com mau comportamento dos estudantes (Tavares; Pietrobom, 2016). Contudo, similarmente aos resultados apresentados por Tavares e Pietrobom (2016), uma maior qualificação dos docentes parece não revelar uma habilidade de reduzir o comportamento agressivo contra a propriedade. Esse mesmo resultado foi observado em relação às estimativas contra a vida, conforme a Tabela 3 .

Em relação às variáveis de renda, verifica-se que no caso de violência contra a vida, a renda é uma variável importante para explicar diferentes 
probabilidades de violência escolar, uma vez que os coeficientes mostram que professores que recebem mais de 4 salários mínimos tendem a relatar menos casos de violência.

Tabela 3 Logit: probabilidade de o professor ser vítima na escola em 2017, Brasil

\begin{tabular}{|c|c|c|c|c|}
\hline \multirow[t]{2}{*}{ Variáveis } & \multicolumn{2}{|c|}{ Contra a vida } & \multicolumn{2}{|c|}{ Contra a propriedade } \\
\hline & (1) & (2) & (1) & (2) \\
\hline \multicolumn{5}{|l|}{ Característica do professor } \\
\hline Mulher & $0,709 * * *$ & $0,690 * * *$ & $1,134^{* *}$ & $1,127^{*}$ \\
\hline Não branco & $0,920 * *$ & 0,978 & $1,091^{*}$ & 1,094 \\
\hline Maior que graduação & 0,967 & 0,988 & 0,989 & 1,055 \\
\hline Maior que 4 salários mínimos & $0,854^{* * *}$ & $0,855^{* * *}$ & 1,036 & 0,973 \\
\hline Carga horária superior a 40 horas & 1,006 & 0,991 & $1,123^{* *}$ & 1,063 \\
\hline Experiência superior a 2 anos & 1,034 & 1,039 & 1,138 & 1,136 \\
\hline Aplicação de normas & $0,677^{* * *}$ & $0,680^{* * *}$ & $0,707^{* * *}$ & $0,720^{* * *}$ \\
\hline Ambiente desestruturado & $4,143^{* * *}$ & $3,941^{* * *}$ & $3,608^{* * *}$ & $3,391^{* * *}$ \\
\hline Ambiente violento & $4,203^{* * *}$ & $4,265^{* * *}$ & $3,866^{* * *}$ & $3,830 * * *$ \\
\hline $\mathrm{N}^{\circ}$ de alunos na sala & 1,002 & $1,007^{* *}$ & $1,016^{* * *}$ & $1,012^{* * *}$ \\
\hline \multicolumn{5}{|l|}{ Característica do aluno } \\
\hline Homens & - & $1,003^{*}$ & - & $1,004^{* *}$ \\
\hline Não branco & - & 1,002 & - & $1,003^{* *}$ \\
\hline Reside com pais & - & $0,995^{* * *}$ & - & $0,993^{* * *}$ \\
\hline Ensino médio ou superior (mãe) & - & $0,995^{* * *}$ & - & 0,998 \\
\hline Reprovação & - & 1,001 & - & 1,001 \\
\hline Abandono & - & $1,007^{*}$ & - & 1,003 \\
\hline Controle localidade e diretor & Não & Sim & Não & Sim \\
\hline $\mathrm{N}^{\circ}$ observações & 38.263 & 38.263 & 38.263 & 38.263 \\
\hline Pseudo $R^{2}$ & 0,1105 & 0,1152 & 0,0799 & 0,0897 \\
\hline Wald $X^{2}$ & $2.334,49$ & $2.419,98$ & $1.299,46$ & $1.423,06$ \\
\hline
\end{tabular}

Fonte: Elaboração própria, com base nos dados da Prova Brasil (2017).

Nota: $P$-valores entre parênteses, em que ${ }^{* * *} \rho \leq 0,01$, ${ }^{* *} 0,01<\rho \leq 0,05,{ }^{*} 0,05<\rho \leq 0,10$. Erros-padrão robustos utilizados com cluster por escolas. A descrição das variáveis está apresentada na Tabela A1. As estimações também foram controladas pelo ano que o professor ministrava suas aulas $\left(5^{\circ}, 9^{\circ}\right.$, do ensino fundamental, $e 3^{\circ}$ ano do ensino médio).

A experiência do professor é outra variável que pode exercer fundamental importância na redução da violência escolar. Entretanto, os resultados encontrados não são significativos em nenhuma das estimativas. Berg e Cor- 
nell (2016) discorrem, por exemplo, que professores com mais experiência relatam menos vitimização e apresentam maior sentimento de segurança na escola, devido, principalmente, obterem maior respeito junto aos alunos, por demonstrarem mais confiança e parecerem menos vulneráveis do que os profissionais menos experientes naquele ambiente.

Outra questão importante diz respeito à capacidade de organização e tomada de decisão da direção da escola. Nesse aspecto, a introdução da variável aplicação das normas é pertinente ao avaliar a importância das normas administrativas. Os resultados indicam que a percepção do professor sobre a aplicação das normas na escola é um fator que reduz a violência escolar, como fundamentado por Huang et al. (2017).

Adicionalmente, verifica-se também que o ambiente escolar e o acesso às armas brancas ou de fogo podem aumentar consideravelmente a probabilidade de relatos de violência, pois as variáveis "Desestruturada" e "Violenta" foram significativas em ambas as especificações e apresentaram coeficientes extremamente elevados. Esse resultado demonstra que maiores chances de violência contra os professores estão diretamente associadas a ambientes escolares negativos (Espelage et al., 2013).

Através da análise das características médias dos alunos em cada classe, reforça-se a ideia de que variáveis relacionadas ao ambiente são importantes para afetar a probabilidade de um professor reportar ser vítima de crimes no âmbito escolar. Dessa forma, observa-se que turmas com maior quantidade de alunos do sexo masculino tendem a causar aumento na probabilidade de relatos contra crimes. Esse resultado é consistente com outras aplicações empíricas internacionais (Khoury-Kassabri et al., 2009; Jaureguizar et al., 2013; Chen; Astor, 2008; Agnich; Miyazaki, 2013).

Destaque deve ser dado para a variável que faz referência residir com ambos os pais. Como verificado, quanto maior a média de alunos que residem com seus pais, menores são as chances de o professor relatar tanto violência física como crimes contra a propriedade. Isso indica que a presença dos pais junto a essas crianças e adolescentes, uma proxy para estrutura e ambiente familiar, é um importante determinante para diminuição de relatos de violência (Smith; Sandhu, 2004; Schochet et al., 2008; Ibabe et al., 2013).

Em relação à escolaridade da mãe, os resultados encontrados apontam uma associação negativa entre o nível educacional da mãe e violência contra a vida, sugerindo que a propensão de os alunos se envolverem em aten- 
tados contra a vida é menor quanto maior for a escolaridade da mãe, ainda que tal variável não tenha sido estatisticamente significativa em crimes contra a propriedade. Assim, demonstra-se a importância do papel da família, ou seja, questões familiares sobre a violência são deveras importantes conforme evidenciado também por Ibabe et al. (2013).

Além disso, conforme fundamentado por Lowe et al. (2020), variáveis relacionadas à localidade possuem grande representatividade na violência escolar, uma vez que distintas regiões podem apresentar diferenças significativas em termos socioeconômicos e demográficos. Dessa forma, foram incluídas variáveis de região geográfica como controle.

A segunda análise, através do modelo Logit Multinomial, objetiva verificar como a violência e demais variáveis explicativas e de controle impactam sobre o conteúdo desenvolvido em sala de aula. Assim como no modelo anterior, foram estimadas duas especificações econométricas, uma verificando o conteúdo desenvolvido em sala de aula de $60 \%$ a $80 \%$ em comparação a menos de $60 \%$, e outra de mais de $80 \%$ em comparação a menos de $60 \%$. O objetivo é verificar se a constatação de Espelage et al. (2013) e Elliott et al. (1998) de que o desempenho em sala de aula, diante da violência, possa ser prejudicado. Novamente, em cada especificação foram realizadas estimações com e sem os controles adicionais de características da sala e da escola (ambiente escolar), em que os coeficientes das regressões são representados pelas razões de risco relativo, conforme Tabela 4.

Dos resultados encontrados, verifica-se que as mulheres possuem maiores chances de ministrar entre $60 \%$ e $80 \%$ do conteúdo, em comparação com os homens. Bem como, são as mulheres que possuem maiores chances de relatarem ministrar mais de $80 \%$ do conteúdo em sala, em comparação com o grupo que relatou ministrar menos de $60 \%$ do conteúdo. Contudo, os resultados parecem ser sensíveis, uma vez que ao se incluírem controles adicionais, essa variável deixa de ser estatisticamente significativa.

Em relação à experiência do professor, encontrou-se uma associação positiva, ou seja, quanto maior a experiência do professor, maiores são as chances de o professor ministrar a maior parte do conteúdo proposto, porém tais resultados não foram estatisticamente significativos.

Professores autodeclarados não brancos possuem menor probabilidade de ministrar mais conteúdo em sala de aula. A renda, o nível de escolaridade e a carga horária do professor são outras variáveis que merecem atenção nas análises, pois influenciam de forma relevante o conteúdo desenvolvido 
em aula pelo professor. As estimativas apontaram uma associação positiva para as três variáveis.

Tabela 4 Logit Multinomial: conteúdo desenvolvido em aula em 2017, Brasil

\begin{tabular}{|c|c|c|c|c|}
\hline \multirow[t]{2}{*}{ Variáveis } & \multicolumn{2}{|c|}{$\begin{array}{r}\text { De } 60 \% \text { a } 80 \% \text { vs } \\
\text { menos de } 60 \%\end{array}$} & \multicolumn{2}{|c|}{$\begin{array}{r}\text { Mais de } 80 \% \text { vs } \\
\text { menos de } 60 \%\end{array}$} \\
\hline & (1) & (2) & (1) & (2) \\
\hline \multicolumn{5}{|l|}{ Característica do professor } \\
\hline Mulher & $1,083^{* *}$ & 1,025 & $1,132 * * *$ & 0,981 \\
\hline Não branco & $0,757^{* * *}$ & $0,855^{* * *}$ & $0,560 * * *$ & $0,766^{* * *}$ \\
\hline Maior que graduação & $1,125^{* * *}$ & $1,132^{* * *}$ & $1,134 * * *$ & $1,178^{* * *}$ \\
\hline Maior que 4 salários mínimos & $1,131^{* * *}$ & $1,117^{* * *}$ & $1,285^{* * *}$ & $1,245^{* * *}$ \\
\hline Carga horária superior a 40 horas & $1,127^{* * *}$ & $1,091^{* *}$ & $1,307^{* * *}$ & $1,171^{* * *}$ \\
\hline Experiência superior a 2 anos & 1,101 & 1,133 & 1,099 & 1,153 \\
\hline Aplicação de normas & $1,490 * * *$ & $1,452^{* * *}$ & $2,115^{* * *}$ & $1,991^{* * *}$ \\
\hline Ambiente desestruturado & $0,786^{* * *}$ & $0,823^{* * *}$ & $0,639 * * *$ & $0,699 * * *$ \\
\hline Ambiente violento & $0,767^{* * *}$ & $0,763^{* * *}$ & $0,639 * * *$ & $0,644^{* * *}$ \\
\hline $\mathrm{N}^{\circ}$ de alunos na sala & 1,002 & 0,997 & 1,004 & $0,994^{* *}$ \\
\hline Contra a vida & $0,772^{* * *}$ & $0,777^{* * *}$ & $0,667^{* * *}$ & $0,686^{* * *}$ \\
\hline Contra a Propriedade & $0,799 * * *$ & $0,795^{* * *}$ & $0,771^{* * *}$ & $0,763^{* * *}$ \\
\hline \multicolumn{5}{|l|}{ Característica do aluno } \\
\hline Homens & - & $0,996^{* * *}$ & - & $0,994 * * *$ \\
\hline Não branco & - & 0,999 & - & $0,994^{* * *}$ \\
\hline Reside com pais & - & $1,005^{* * *}$ & - & $1,009 * * *$ \\
\hline Ensino médio ou superior (mãe) & - & $1,005^{* * *}$ & - & $1,011^{* * *}$ \\
\hline Reprovação & - & $0,994^{* * *}$ & - & $0,989 * * *$ \\
\hline Abandono & - & $0,994^{* *}$ & - & $0,986^{* * *}$ \\
\hline Controle localidade e diretor & Não & $\operatorname{Sim}$ & Não & Sim \\
\hline $\mathrm{N}^{\circ}$ observações & 38.263 & 38.263 & 38.263 & 38.263 \\
\hline Pseudo $R^{2}$ & 0,0269 & 0,0473 & 0,0269 & 0,0473 \\
\hline Wald $X^{2}$ & $1.750,13$ & $2.951,80$ & $1.750,13$ & $2.951,80$ \\
\hline
\end{tabular}

Fonte: Elaboração própria, com base nos dados da Prova Brasil (2017).

Nota: $P$-valores entre parênteses, em que ${ }^{* * *} \rho \leq 0,01,{ }^{* *} 0,01<\rho \leq 0,05,{ }^{*} 0,05<\rho \leq 0,10$. Erros-padrão robustos utilizados com cluster por escolas. A descrição das variáveis está apresentada na Tabela A1. As estimações também foram controladas pelo ano que o professor ministrava suas aulas $\left(5^{\circ}, 9^{\circ}\right.$, do ensino fundamental, e $3^{\circ}$ ano do ensino médio). 
Por fim, analisa-se o impacto do ambiente violento e da vitimização sobre o conteúdo desenvolvido. Como esperado, lecionar em ambientes em que há relatos de presença de armas, alunos alcoolizados ou sob efeito de drogas reduz as chances de o docente relatar ministrar o conteúdo programado, confirmando a relação negativa entre desempenho escolar e violência apontada por Teixeira e Kassouf (2015). Além disso, professores que relataram terem sido vítimas de crimes contra a propriedade ou violência física também apresentam menores chances de ministrar o conteúdo programado em classe, em todas as especificações analisadas, sendo todos os resultados estatisticamente significativos. Esse resultado é uma das diversas consequências que a violência escolar proporciona à comunidade escolar (Galand et al., 2004), demonstrando a urgência de medidas para atenuação dessa realidade brasileira.

Para verificar o ambiente em sala de aula, variáveis que consideram as características dos estudantes de cada classe também foram empregadas. Percebe-se que quanto maior a média de alunos do sexo masculino ou de alunos autodeclarados não brancos em uma classe menores são as chances de um professor ministrar o conteúdo. Classes de aula com maiores médias de alunos que residem com os pais são aquelas em que o professor possui maior chance de relatar ministrar maior porcentagem do conteúdo programado. Isso ocorre dada a importância do apoio dos pais no desempenho escolar dos alunos (Vettenburg, 2002).

Em relação à reprovação e ao abandono, o comportamento esperado é de que, em turmas de alunos com defasagem idade-série ou que abandonaram os estudos, o conteúdo seja mais lentamente aplicado, em vista das dificuldades encontradas pelos alunos e professores, similarmente ao estudo de Gerberich et al. (2011). Os resultados obtidos dão subsídio a essa interpretação, uma vez que foi encontrada uma relação negativa entre taxa de reprovação e conteúdo ministrado, assim como entre taxa de abandono e conteúdo ministrado.

Os resultados apresentados mostram claramente a gravidade dos problemas de violência contra os professores nas escolas de ensino fundamental e médio no Brasil. A violência nas escolas gera inúmeros problemas para toda a comunidade escolar, com externalidades negativas para todo o sistema educacional brasileiro. Nesse sentido, emerge a necessidade de discutir soluções estratégicas para prevenção da violência nas escolas, as quais devem ser implementadas por todos os agentes envolvidos na edu- 
cação dos jovens - pais, educadores, prefeituras, governos federal, estaduais e municipais.

No âmbito das escolas, inúmeras estratégias para aproximação da comunidade escolar podem ser implantadas, conscientizando os alunos sobre a importância da preservação do patrimônio público. Além disso, de acordo com os resultados do presente estudo, a aplicação de regras parece ser importante, uma vez que muitos professores sentem falta de apoio administrativo, incluindo falta de liderança, ações escolares ineficazes e desorganização escolar (Mcmahon et al., 2017; Moon et al., 2019). Ao envolver pais e alunos na resolução dos conflitos internos das escolas, a solução para a redução da violência ganha aliados fundamentais, uma vez que esse envolvimento eleva o potencial de aprendizado dos alunos para além dos conhecimentos restritos às ementas educacionais. Esse envolvimento é fundamental para que os professores possam atuar ativamente em sala de aula e pode ser observado pelo impacto negativo entre residir com os pais e a violência escolar (Vettenburg, 2002).

Contudo, é fundamental visualizar a importância das ações dos professores, diretores e agentes escolares para a redução da violência nas escolas que, em alguns casos, são os primeiros a presenciarem uma mudança de comportamento dos jovens. No entanto, os ensinamentos sobre gestão comportamental e mediação de conflitos não são prioridades dentro do programa de preparação dos agentes escolares (Espelage et al., 2013). Assim, deve-se existir uma preocupação das autoridades governamentais na preparação dos agentes para mediação dos conflitos, compartilhando experiências e conhecimentos adquiridos nas escolas do Brasil. Além disso, é necessário que haja um acompanhamento das lideranças das escolas sobre o bem-estar emocional dos professores, uma vez que sintomas de ansiedade, depressivos e somáticos reportados pelos professores estão positivamente associados aos relatos de violência escolar (Galand et al., 2007).

Nesse contexto, a violência escolar é uma questão que está sendo analisada em todo o mundo e deve se tornar parte corriqueira das avaliações institucionais, com respectivas estratégias de interversões e medidas corretivas (Longobardi et al., 2018). Uma alternativa de fácil execução é a elaboração e divulgação de indicadores de violência escolar a partir de base de dados já coletadas regularmente (como os dados da Prova Brasil), uma vez que facilitaria o acompanhamento das autoridades governamentais, assim como de toda a comunidade acadêmica. 


\section{Considerações finais}

O presente estudo busca avaliar quais são as características que influenciam a probabilidade de um professor relatar ter sido ou não vítima violência no Brasil. Para tal, foi empregado o modelo econométrico Logit. Avalia-se, também, se a violência relatada impacta sobre o conteúdo desenvolvido pelos professores em sala de aula, através de um modelo Logit Multinomial. Para alcançar esses objetivos foram utilizados os dados fornecidos pelo SAEB/Prova Brasil de 2017.

A fração de professores exposta a violência (contra vida ou contra a propriedade) dentro do ambiente escolar no último ano chama a atenção: $8,24 \%$ dos professores analisados sofreram crimes contra propriedade, enquanto $5,27 \%$, violência de crime contra a vida. Esses números, apesar de interpretação relativa, não são capazes de retratar o tamanho do problema, tão pouco as suas causas, uma vez que podem ser qualitativamente distintos e acarretar prejuízos físicos, financeiros e psicológicos profundos aos professores.

Através da análise econométrica se identificou que os principais determinantes da maior chance de relatos de vitimização dentro do ambiente escolar estão relacionados ao ambiente de trabalho desse professor. Por exemplo, a presença de alunos sob efeito de droga/álcool e/ou e portando arma branca/fogo no ambiente escolar aumentaram consideravelmente a probabilidade de relatos de violência contra o professor, seja ela contra vida ou contra a propriedade.

Na segunda parte, ao investigar fatores que impactam o conteúdo desenvolvido em sala de aula pelos professores, novamente, um ambiente escolar comprometido pela violência se mostra prejudicial. Em magnitudes similares, professores que relataram lecionar em um ambiente considerado violento ou desestruturado e professores que indicaram terem sido vítimas de crimes contra a vida ou a propriedade, possuem menores chances de apresentar o conteúdo programado.

Os resultados apontam para a gravidade do problema, em que professores vítimas ou em ambientes desfavoráveis ao ensino não são capazes de ministrar o conteúdo básico programado. Dessa forma, é necessária a elaboração de políticas públicas com o envolvimento de todos os agentes interessados na educação dos alunos (pais, professores, diretores e governos municipais, estaduais e federal). Tais políticas devem ser desenhadas 
levando em consideração a localização geográfica e a realidade socioeconômica em que a escola e seus participantes estão inseridos.

Por fim, destaca-se a necessidade de continuação da pesquisa para anos posteriores para confirmação desses resultados, pois algumas limitações são observadas. Professores que foram vítimas de atos de violência podem não estar presentes no questionário (mudança de profissão). Além disso, apesar de identificar menor probabilidade de ministrar o conteúdo programado, não foi analisada a qualidade do conteúdo lecionado, que também pode ter sido afetada pela violência. Fatores limitadores nesta pesquisa, mas que podem indicar uma importância ainda maior desse tema de estudo.

\section{Referências}

AGNICH, L. E.; MIYAZAKI, Y. A cross-national analysis of principals' reports of school violence. International Criminal Justice Review, v. 23, n. 4, p. 378-400, 2013.

BECKER, K. L.; KASSOUF, A. L. Violência nas escolas públicas brasileiras: uma análise da relação entre o comportamento agressivo dos alunos e o ambiente escolar. Nova Economia, v. 26, n. 2 , p. $653-677,2016$.

BERG, J. K.; CORNELL, D. Authoritative school climate, aggression toward teachers, and teacher distress in middle school. School Psychology Quarterly, v. 31, n. 1, p. 122-139, 2016.

CAMERON, A. C.; TRIVEDI, P. K. Microeconometrics: methods and applications. Cambridge; New York: Cambridge University Press, 2005.

CARVALHO, M. G.; OLIVEIRA, G. F.; CARITA, A. Representações da violência por professores. Id on Line Revista Multidisciplinar e de Psicologia, v. 10, n. 29, p. 7-22, 2016.

CHEN, J.; ASTOR, R. A. Students' reports of violence against teachers in Taiwanese schools. Journal of School Violence, v. 8, n. 1, p. 2-17, 2008.

DUARTE, R. G. Os determinantes da rotatividade dos professores no Brasil: uma análise com base nos dados do SAEB 2003. Tese (Doutorado) - Universidade de São Paulo, São Paulo, 2009.

ELLIOTT, D. S.; HAMBURG, B. A.; WILLIAMS, K. R. (Ed.). Violence in American schools: A new perspective. New York: Cambridge University Press, 1998.

ESPELAGE, D.; ANDERMAN, E. M.; BROWN, V. E.; JONES, A.; LANE, K. L.; MCMAHON, S. D.; REDDY, L. A.; REYNOLDS, C. R. Understanding and preventing violence directed against teachers: Recommendations for a national research, practice, and policy agenda. American Psychologist, v. 68, n. 2, p. 75-87, 2013.

GALAND, B.; PHILIPPOT, P.; PETIT, S.; BORN, M.; BUIDIN, G. Regards croisés sur les phénomènes de violence en milieu scolaire: élèves et équipes éducatives. Revue des Sciences de l'Éducation, v. 30, n. 3, p. 465-486, 2004. 
GALAND, B.; LECOCQ, C.; PHILIPPOT, P. School violence and teacher professional disengagement. British Journal of Educational Psychology, v. 77, n. 2, p. 465-477, 2007.

GERBERICH, S. G.; NACHREINER, N. M.; RYAN, A. D.; CHURCH, T. R.; MCGOVERN, P. M.; GEISSER, M. S.; MONGIN, St. J.; WATT, G. D.; FEDA, D. M.; SAGE, S. K.; PINDER, E. D. Violence against educators: A population-based study. Journal of Occupational and Environmental Medicine, v. 53, n. 3, p. 294-302, 2011.

GLAESER, E. L.; SACERDOTE, B. Why is there more crime in cities? Journal of Political Economy, v. 107, n. S6, p. S225-S258, 1999.

GREENE, W. H. Econometric Analysis. Pearson, 7ed., 2011.

HUANG, F. L.; EDDY, C. L.; CAMP, E. The role of the perceptions of school climate and teacher victimization by students. Journal of Interpersonal Violence, v. 35, p. 5.526-5.551, 2017.

IBABE, I.; JAUREGUIZAR, J.; BENTLER, P. M. Protective factors for adolescent violence against authority. The Spanish Journal of Psychology, v. 16, p. 1-13, 2013.

INEP. Indicador de nivel socioeconômico das escolas de educação básica (inse) em 2015. Disponível em: <http://download.inep.gov.br/informacoes_19estatisticas/indicadores_educacionais/2015/nota_tecnica/nota_tecnica_inep_inse_2015.pdf>. Acesso em: 10 mar. 2019.

JAUREGUIZAR, J.; IBABE, I.; STRAUS, M. A. Violent and prosocial behavior by adolescents toward parents and teachers in a community sample. Psychology in the Schools, v. 50, n. 5, p. 451-470, 2013.

KHOURY-KASSABRI, M.; ASTOR, R. A.; BENBENISHTY, R. Middle Eastern adolescents' perpetration of school violence against peers and teachers: A cross-cultural and ecological analysis. Journal of Interpersonal Violence, v. 24, n. 1, p. 159-182, 2009.

LONGOBARDI, C.; BADENES-RIBERA, L.; FABRIS, M. A.; MARTINEZ, A.; MCMAHON, S. D. Prevalence of student violence against teachers: A meta-analysis. Psychology of Violence, v. 9, n. 6, p. 596-610, 2018.

LOWE, E.; PICKNOLL, D.; CHIVERS, P.; FARRINGDON, F.; RYCROFT, P. Teacher-directed violence by students in Western Australia: An exploratory study. Issues in Educational Research, v. 30, n. 1, p. 187-202, 2020.

MCMAHON, S. D.; REAVES, S.; MCCONNELL, E. A.; PEIST, E.; RUIZ, L. The ecology of teachers' experiences with violence and lack of administrative support. American Journal of Community Psychology, v. 60, n. 3-4, p. 502-515, 2017.

MELANDA, F. N.; SANTOS, H. G.; SALVAGIONI, D. A. J.; MESAS, A. E.; GONZÁLEZ, A. D.; ANDRADE, S. M. Violência física contra professores no espaço escolar: análise por modelos de equações estruturais. Cadernos de Saúde Pública, v. 34, n. 5, p. 1-11, 2018.

MILLER, M. D.; BROWNELL, M. T.; SMITH, S. W. Factors that predict teachers staying in, leaving, or transferring from the special education classroom. Exceptional Children, v. 65, n. 2, p. 201-218, 1999.

MONTEIRO, J.; ROCHA, R. Drug battles and school achievement: Evidence from Rio de Janeiro's favelas. Review of Economics and Statistics, v. 99, n. 2, p. 213-228, 2017.

MOON, B.; MORASH, M.; MCCLUSKEY, J. Student violence directed against teachers: Victimized teachers' reports to school officials and satisfaction with school responses. Journal 
of Interpersonal Violence, p. 1-20, 2019.

NEWMAN, K. S.; FOX, C.; HARDING, D.; MEHTA, J.; ROTH, W. Rampage: The social roots of school shootings. Hachette UK, 2008.

OCDE. TALIS 2013 Results: An International Perspective on Teaching and Learning. TALIS, OECD Publishing. Paris, 2014. Disponível em: <http://www.oecd.org/education/school/ TALIS-technical-report-2013.pdf >. Acesso em: 1 fev. 2019.

PROVA BRASIL. Microdados do Sistema Nacional de Avaliação da Educação Básica de 2017. Disponível em: <http://download.inep.gov.br/microdados/microdados_saeb_2017.zip>. Acesso em: 21 mar. 2019.

ROCHA, K. M. M.; FARIAS, G. M.; GURGEL, A. K. C.; COSTA, I. K. F.; FREITAS, M. C. S.; SOUZA, A. A. M. Violência na escola vivida por professores, funcionários e diretores. Revista da Rede de Enfermagem do Nordeste, v. 13, n. 5, p. 1.034-1.044, 2012.

SHOCHET, I. M.; HOMEL, R.; COCKSHAW, W. D.; MONTGOMERY, D. T. How do school connectedness and attachment to parents interrelate in predicting adolescent depressive symptoms? Journal of Clinical Child \& Adolescent Psychology, v. 37, n. 3, p. 676-681, 2008.

SILVA, O. G.; NAVARRO, E. C. A relação professor-aluno no processo ensino-aprendizagem. Revista Eletrônica Interdisciplinar, v. 2, n. 8, p. 95-100, 2012.

SILVA, E. N.; ROSA, E. C. de. Professores sabem o que é bullying? Um tema para a formação docente. Revista Semestral da Associação Brasileira de Psicologia Escolar e Educacional, v 17, n. 2, p. 329-338, 2013.

SMITH, D. C.; SANDHU, D. S. Toward a positive perspective on violence prevention in schools: Building connections. Journal of Counseling \& Development, v. 82, n. 3, p. 287-293, 2004.

TAVARES, P. A.; PIETROBOM, F. C. Fatores associados à violência escolar: evidências para o Estado de São Paulo. Estudos Econômicos (São Paulo), v. 46, n. 2, p. 471-498, 2016.

TEIXEIRA, E.; KASSOUF, A. L. Impacto da violência nas escolas paulistas sobre o desempenho acadêmico dos alunos. Economia Aplicada, v. 19, n. 2, p. 221-240, 2015.

VETTENBURG, N. Unsafe feelings among teachers. Journal of School Violence, v. 1, n. 4, p. 33-49, 2002.

WILSON, C. M.; DOUGLAS, K. S.; LYON, D. R. Violence against teachers: Prevalence and consequences. Journal of Interpersonal Violence, v. 26, n. 12, p. 2.353-2.371, 2011.

\section{Sobre os autores}

Wander Plassa - wanderplassa@gmail.com

Centro de Ciências Sociais Aplicadas, Universidade Estadual do Paraná, Apucarana, PR, Brasil.

ORCID: https://orcid.org/0000-0003-3302-5979.

Pietro André Telatin Paschoalino - pietropasch@gmail.com

Universidade Estadual de Maringá, Maringá, PR, Brasil.

ORCID: https://orcid.org/0000-0002-3259-1285.

Luan Vinicius Bernardelli - luanbernardelli@gmail.com

Centro de Ciências Sociais Aplicadas, Universidade Estadual do Paraná, Apucarana, Paraná, PR, Brasil.

ORCID: https://orcid.org/0000-0003-1410-2318. 
Os autores agradecem os comentários realizados pelos pareceristas anônimos e ao editor da revista Nova Economia, que contribuíram para melhoria e melhor clareza do artigo. O presente trabalho foi realizado com apoio da Coordenação de Aperfeiçoamento de Pessoal de Nível Superior (CAPES) - Código de Financiamento 001.

\section{Sobre $\mathrm{o}$ artigo}

Recebido em 16 de outubro de 2019. Aprovado em 01 de junho de 2020. 


\section{APÊNDICE}

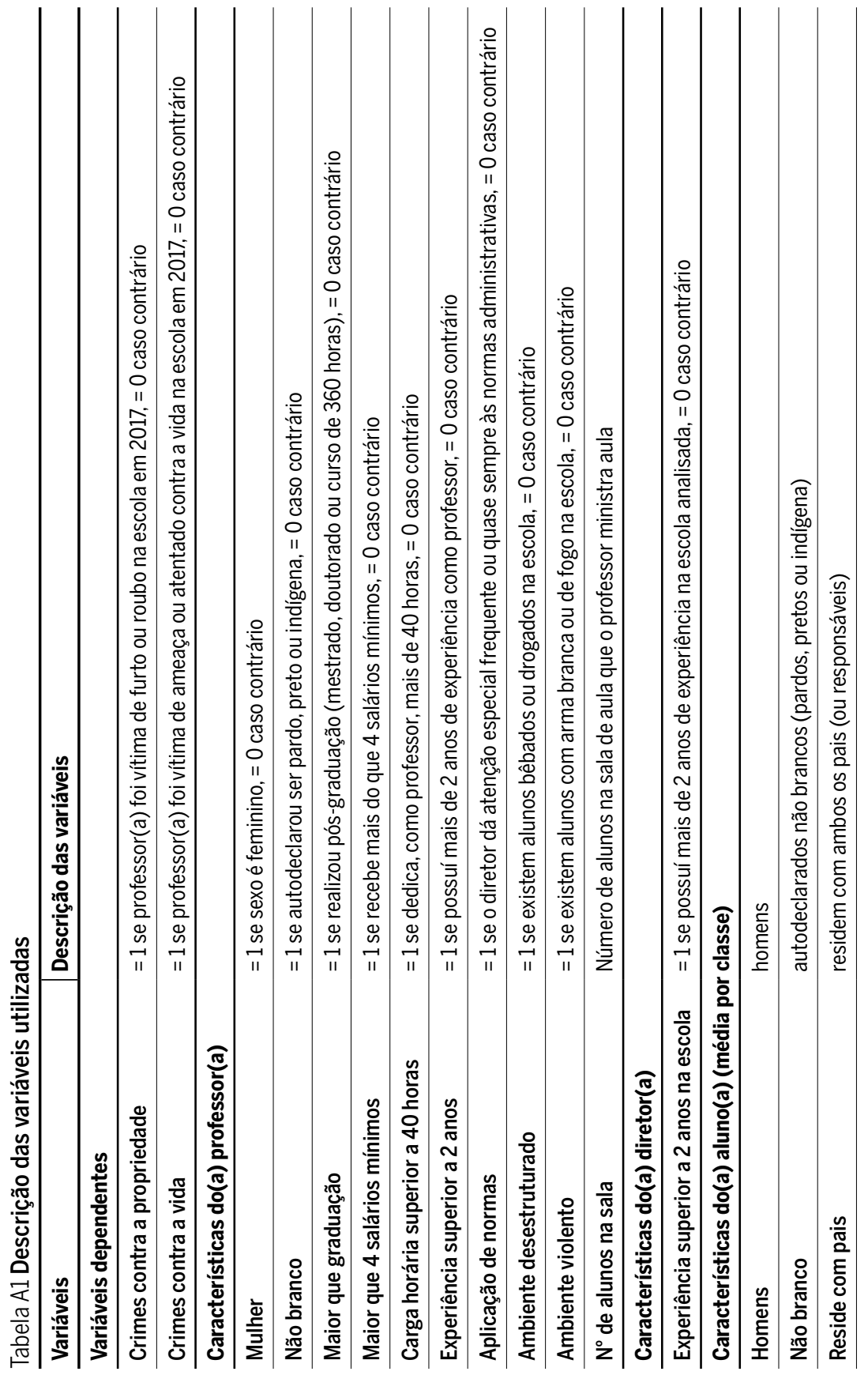




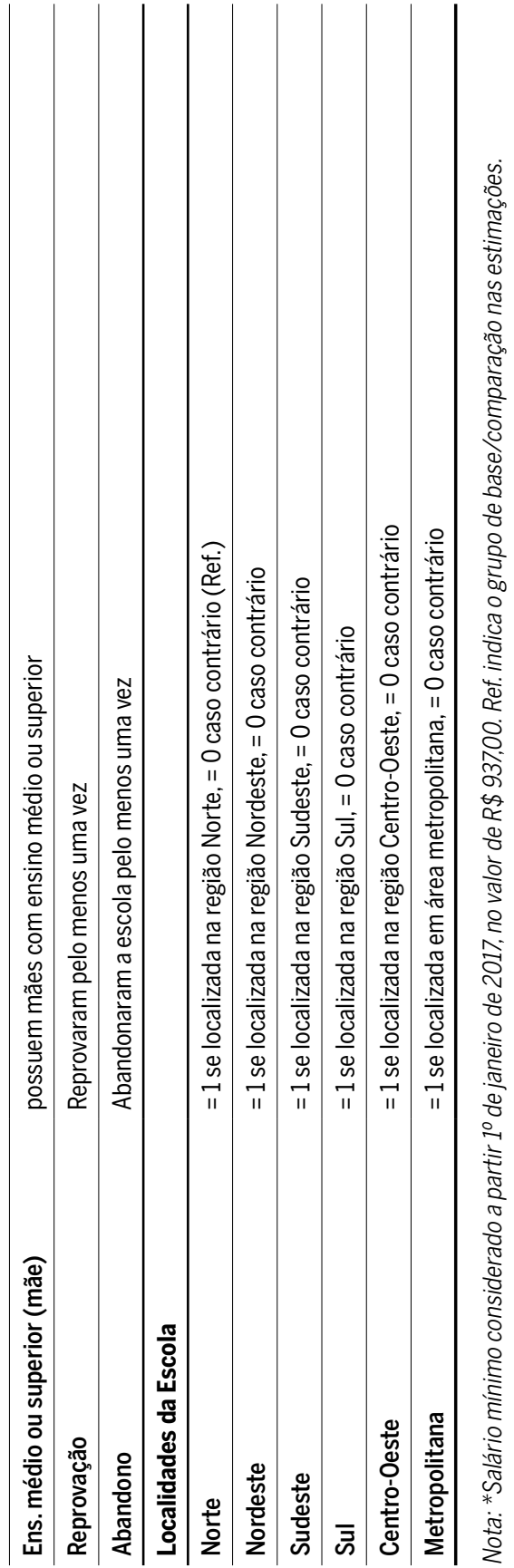

